\title{
MODELS OF MICROBIAL DORMANCY IN BIOFILMS AND PLANKTONIC CULTURES*
}

\author{
BRUCE P. AYATI ${ }^{\dagger}$ AND ISAAC KLAPPER $\ddagger$
}

\begin{abstract}
We present models of dormancy in planktonic cultures and in biofilm, and a new numerical technique for solving the model equations. We use this modeling framework to examine the relative advantage of short dormancy versus long dormancy times in planktonic cultures and biofilms under some basic assumptions. Simulations and asymptotic analyses indicate that in planktonic batch cultures and in chemostats, live biomass is maximized by the fastest possible exit from dormancy. The lower limit of time to reawakening is thus perhaps governed by physiological, biochemical, or other constraints within the cells. In biofilm we see, in contrast, that the slower waker may have an advantage over the faster waker.
\end{abstract}

Key words. Biofilm, physiological structure, dormancy, bacteria.

AMS subject classifications. 35B40, 65M25, 92-04, 92-08, 92C37, 92C50.

\section{Introduction}

Microbial populations, particularly those in biofilms (sessile, matrix encased communities; see [16] for an overview), can contain cells in varying phenotypic or physiological states. An important difference between planktonic (free-swimming) and biofilm environments is that the former is generally well-mixed whereas the latter is unmixed and spatially heterogeneous as a result of their self-generated spatially variable environments $[22,26]$. Models that include phenotypic and physiological variation can aid in understanding biofilms and planktonic cultures, and the differences between them. Structured population methods are particularly well-suited for tracking internal states.

Here we consider one type of physiological state - dormancy (possibly related to the phenomenon of persister cells $[6,9,10,17]$ ) - where, in response to an environmental stress, cells differentiate into a protected, slow- or non-growing condition $[8,20]$. Bacteria in planktonic states have been found to revive faster from dormancy than those in a biofilm state [8]. Thus it would seem that dormancy-regulating parameters are subject to influence of environmental variation, at least of the sort found in biofilms.

We present a modeling and simulation framework designed to study dormancy within batch, chemostat, and biofilm population dynamics, in particular with respect to competitiveness. The physiological structure is represented by a continuous variable (see [25] for a broader discussion). Compartmentalized dormancy models have been considered elsewhere $[18,19]$.

These tools are an extension of those discussed in [6] for persistence and senescence, primarily in the numerical methods used to solve the more general physiological structure used in this paper, and we expect they will have wider applicability to descriptions of physiological states in both mixed and unmixed microbial communities. Robust and efficient numerical methods for handling physiological structure may be

*Received: November 9, 2010; accepted (in revised version): July 24, 2011. Communicated by Andrea Bertozzi.

${ }^{\dagger}$ Department of Mathematics and Program in Applied Mathematical \& Computational Sciences, University of Iowa, Iowa City, IA 52242-1419 (bruce-ayati@uiowa.edu).

${ }^{\ddagger}$ Department of Mathematical Sciences and Center for Biofilm Engineering, Montana State University (klapper@math.montana.edu). 
necessary as additional interacting phenotypes are included in the study of complex microbial communities.

We use our modeling tools to gain insight into role and regulation of dormancy in spatially mixed systems (batch and chemostat microbial communities) and unmixed systems (biofilm communities). Our attention is directed to the relative advantage of short dormancy versus long dormancy times in the cases of batch, chemostat, and biofilm states.

This paper is organized as follows. We present models of chemostat and batch cultures, and then derive the biofilm model. We discuss the numerical methods developed for these computations and compute numerical solutions of the model equations for the batch, chemostat, and biofilm cultures. We then provide a study of the convergence of the numerical results. To aid in understanding our computations, we present asymptotic analyses of their long-time behavior. We conclude with a discussion of the implications of our results.

\section{Models of dormancy in chemostat and batch cultures}

We introduce $s \in\left[s_{0}, s^{*}\right]$ to index the dormancy state of individual cells, with $s_{0}$ the value at which cells enter dormancy and $s^{*}$ the value at which cells leave dormancy and become active. Cells progress through dormancy states with "speed" $g(s, c)$, where $c$ is concentration of relevant chemicals (e.g. substrates or antimicrobials); for example, large concentrations of substrates and/or small concentrations of antimicrobials imply larger values of $g$. While dormant, cells do not grow and divide; on the other hand, dormant cells are presumed to be hardier in response to environmental stress.

Let $t \geq 0$ represent time. Let $u_{i}(t)$ represent the density of active cells of species $i, v_{i}(s, t)$ represent the density of dormant cells of species $i$, and $c(t)$ be a vector of substrate chemical species concentrations. Let $u$ and $v$ be vectors containing the active and dormant densities, respectively, of all species. Let the operator $\partial_{y}$ denote partial differentiation in the subscript variable $y$. The active cell populations are modeled by an ordinary differential equation for each $i$ and $t>0$,

$$
\begin{aligned}
\frac{d}{d t} u_{i}(t)=\underbrace{b_{i}(c) u_{i}(t)}_{\text {cell division }} & -\underbrace{\mu_{u_{i}}(c) u_{i}(t)}_{\text {death }} \\
& \quad+\underbrace{g_{i}\left(s^{*}, c\right) v_{i}\left(s^{*}, t\right)}_{\text {exit from dormancy }}-\underbrace{h_{i}(c) u_{i}(t)}_{\text {entrance to dormancy }}-\underbrace{d_{0} u_{i}(t)}_{\text {washout }},
\end{aligned}
$$

where $d_{0}$ is the chemostat dilution rate (roughly, the inverse of the time scale for the chemostat contents to be flushed). The functions $b_{i}$ and $\mu_{u_{i}}$ account for cell division and cell "death" for species $i$. We use "death" as shorthand for all forms of inertness not tied to strategic dormancy on the part of the bacteria. The function $h_{i} \geq 0$ is the dormancy rate of active cells. Let $g_{i} \geq 0$ (as above) and $\mu_{v_{i}} \geq 0$ denote the reactivation and death rates, respectively, of the dormant cells. We use physiologically structured equations for the dormant cell populations,

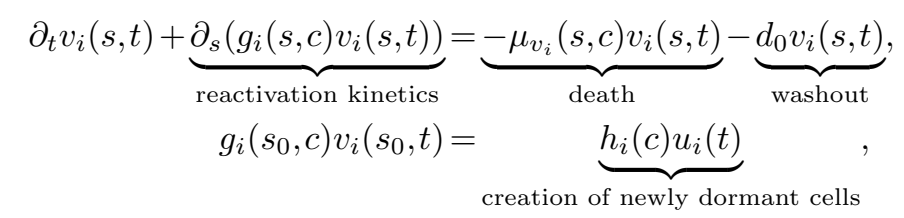




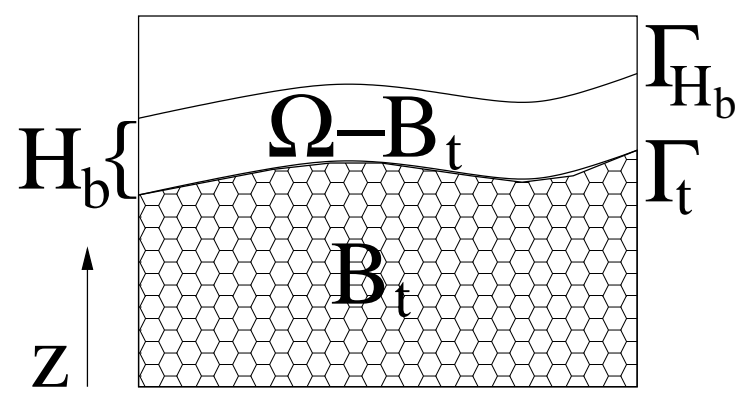

FIG. 3.1. Spatial domains for the biofilm model.

for $s_{0}<s \leq s^{*}$. For the substrate chemicals, we have

$$
\frac{d}{d t} c(t)=-\underbrace{f(c(t), u(t), v(\cdot, t))}_{\text {chemical reactions }}+\underbrace{d_{0}\left(C_{0}(t)-c(t)\right)}_{\text {chemostat turnover }},
$$

where $f$ is the vector of reactions and $C_{0}(t)$ is the input concentration vector from the chemostat tank. Initial conditions are $u(0)=u_{0}, v(0, s)=v_{0}(s)$, and $c(0)=c_{0}$. The model for a batch culture is obtained from (2.1) by setting $d_{0}=0$.

\section{A model of dormancy in a biofilm}

For the biofilm model we remove the chemostat-specific terms and extend the system (2.1) with $i=1$ to include a spatial domain $\Omega$ consisting of stratified subdomains $B_{t}$ for biomass and $\Omega \backslash B_{t}$ for the bulk fluid. There are two moving interfaces in $\Omega$ : $\Gamma_{t}$ separating $B_{t}$ from the rest of $\Omega$, and a bulk-substrate interface $\Gamma_{H_{b}}$ that is a fixed height $H_{b}$ above $\Gamma_{t}$. The biofilm rests on a surface, denoted by a lower boundary $\Gamma_{B}$. The spatial domains are illustrated in Figure 3.1. The active and dormant cell populations, and the chemical concentrations, now depend on $\mathbf{x} \in \Omega$.

Exterior (to the biofilm) flow is neglected here. The principle effects of fluid flow, such as mechanical stress and spatial variation of substrate concentrations in the streamwise direction, are not central to those particular dormancy issues we investigate.

Unlike the planktonic models presented above, we need to track explicitly the fully inert (non-resuscitative) cell biomass as well as the dormant cell biomass since they both affect volume fraction calculations. We denote fully inert cells by $w$. For simplicity we neglect any lysis on the part of those fully inert cells that might be necrotic.

Conservation of biomass yields, for $t>0$ and $s_{0}<s \leq s^{*}$,

$$
\begin{aligned}
\partial_{t} u(\mathbf{x}, t)+\nabla \cdot \mathbf{J}_{u}=b(c) u(\mathbf{x}, t)-\mu_{u}(c) u(\mathbf{x}, t)+g\left(s^{*}, c\right) v\left(\mathbf{x}, s^{*}, t\right)-h(c) u(\mathbf{x}, t) & \\
\partial_{t} v(\mathbf{x}, s, t)+\partial_{s}(g(s, c) v(\mathbf{x}, s, t))+\nabla \cdot \mathbf{J}_{v} & =-\mu_{v}(s, c) v(\mathbf{x}, s, t) \\
g\left(s_{0}, c\right) v\left(\mathbf{x}, s_{0}, t\right) & =h(c) u(\mathbf{x}, t) \\
\partial_{t} w(\mathbf{x}, t)+\nabla \cdot \mathbf{J}_{w} & =\mu_{u}(c) u(\mathbf{x}, t)+\int_{s_{0}}^{s^{*}} \mu_{v}(s, c) v(\mathbf{x}, s, t) d s \\
\partial_{t} c(\mathbf{x}, t)+\nabla \cdot \mathbf{J}_{c} & =f(c(t), u(\mathbf{x}, t), v(\mathbf{x}, \cdot, t))
\end{aligned}
$$


where $\nabla$. denotes divergence in space, $\mathbf{J}_{y}$ denotes the flux of subscript variable $y$, and where we assume appropriate initial conditions and boundary condition on the spatial domains.

Assuming Fick's Law gives $\mathbf{J}_{\mathbf{c}}=-D \nabla c$ with diffusion constant $D$. The substrate masses are also subject to advection, but the velocity is sufficiently slow that we can neglect the advective contribution to substrate flux. Likewise, substrate material diffusion time scales are at least several orders of magnitude shorter than those at which bacteria grow or advect, allowing us to make a quasi-steady-state assumption so that

$$
-D \nabla^{2} c=f .
$$

Let $\vartheta(\mathbf{x}, s, t)$ and $\rho(\mathbf{x}, s, t)$ denote the volume fraction per dormancy state and density per dormancy state relative to volume fraction, respectively, of dormant cells. We assume incompressibility of biomass with $\rho(\mathbf{x}, s, t) \equiv \rho^{*}$ for positive constant $\rho^{*}$. We also assume, based on the fact that the main constituent of all cells is water, that active and fully inert cells have the same incompressibility properties, and the same densities relative to volume fractions, $\rho^{*}$, as dormant cells. We let $\nu(\mathbf{x}, t)$ and $\eta(\mathbf{x}, t)$ denote the volume fractions of active and fully inert cells, respectively, which are related to the density of active and fully inert cells by $u=\rho^{*} \nu$ and $w=\rho^{*} \eta$. We require the biomass volume fractions to total to one so that

$$
\nu(\mathbf{x}, t)+\eta(\mathbf{x}, t)+\int_{s_{0}}^{s^{*}} \vartheta(\mathbf{x}, s, t) d s=1 .
$$

Polymer production depends on activity. We are making the simplifying assumptions that cell growth and polymer production are proportional and that matrix material does not decay. These assumptions have the consequence that density of cells (whether active, dormant or fully inert) and density of polymer matrix are constant.

Assuming that transport of biomass, including dormant cells, is governed by an advective process, with a volumetric flow $\mathbf{u}(\mathbf{x}, t)$, gives the fluxes $\mathbf{J}_{\mathbf{u}}=\rho^{*} u \mathbf{u}, \mathbf{J}_{\mathbf{v}}=$ $\rho^{*} v \mathbf{u}$, and $\mathbf{J}_{\mathbf{w}}=\rho^{*} w \mathbf{u}$. As in [6], we follow $[1,13]$ and assume that the volumetric flow is stress driven according to

$$
\mathbf{u}=-\lambda \nabla p,
$$

where $p(t, \mathbf{x})$ is the pressure, $\lambda>0$ is the Darcy constant, and $p=0$ in $\Omega \backslash B_{t}$. Pressure is determined in order to enforce incompressibility in response to growth and hence (3.4) can be viewed as a balance of growth-induced stress against friction. Other choices of force balance are possible.

Substituting $u=\rho^{*} \nu, \mathbf{J}_{\mathbf{u}}=\rho^{*} u \mathbf{u}, v=\rho^{*} \vartheta, \mathbf{J}_{\mathbf{v}}=\rho^{*} v \mathbf{u}, w=\rho^{*} \eta$, and $\mathbf{J}_{\mathbf{w}}=\rho^{*} w \mathbf{u}$ into Equation (3.1) gives

$$
\begin{aligned}
\partial_{t} \nu(\mathbf{x}, t)+\nabla \cdot(\mathbf{u} \nu)=b(c) \nu(\mathbf{x}, t)-\mu_{v}(c) \nu(\mathbf{x}, t)+g\left(s^{*}, c\right) \vartheta\left(\mathbf{x}, s^{*}, t\right)-h(c) \nu(\mathbf{x}, t), \\
\partial_{t} \vartheta(\mathbf{x}, s, t)+\partial_{s}(g(s, c) \vartheta(\mathbf{x}, s, t))+\nabla \cdot(\mathbf{u} \vartheta)=-\mu_{v}(s, c) \vartheta(\mathbf{x}, s, t), \\
g\left(s_{0}, c\right) \vartheta\left(\mathbf{x}, s_{0}, t\right)=h(c) \nu(\mathbf{x}, t), \\
\partial_{t} \eta(\mathbf{x}, t)+\nabla \cdot(\mathbf{u} \eta)=\mu_{u}(c) \nu(\mathbf{x}, t)+\int_{s_{0}}^{s^{*}} \mu_{v}(s, c) \vartheta(\mathbf{x}, s, t) d s \\
-D \nabla^{2} c=f
\end{aligned}
$$


with appropriate initial conditions and boundary condition on the spatial domains (see (3.10) for details).

Integrating (3.5b) over $s$ gives

$$
\begin{aligned}
\underbrace{\partial_{t}\left(\int_{s_{0}}^{s^{*}} \vartheta(\mathbf{x}, s, t) d s\right)}_{=-\partial_{t} \nu-\partial_{t} \eta} & +g\left(s^{*}, c\right) \vartheta\left(\mathbf{x}, s^{*}, t\right)-g\left(s_{0}, c\right) \underbrace{\vartheta\left(\mathbf{x}, s_{0}, t\right)}_{=h \nu} \\
& +\underbrace{\nabla \cdot\left(\mathbf{u} \int_{s_{0}}^{s^{*}} \vartheta(\mathbf{x}, s, t) d s\right)}_{=\nabla \cdot \mathbf{u}(1-\nu-\eta)}=-\int_{s_{0}}^{s^{*}} \mu_{v}(s, c) \vartheta(\mathbf{x}, s, t) d s
\end{aligned}
$$

Substituting for $-\partial_{t} \nu-\partial_{t} \eta$ yields

$$
\nabla \cdot \mathbf{u}=b(c) \nu
$$

Substituting $\mathbf{u}=-\lambda \nabla p$ gives an equation for the pressure in $B_{t}$,

$$
-\lambda \nabla^{2} p=b(c) \nu
$$

Distributing the divergence operator gives

$$
\begin{aligned}
& \nabla \cdot(\mathbf{u} \nu)=-\lambda \nabla p \cdot \nabla \nu+b(c) \nu^{2}, \\
& \nabla \cdot(\mathbf{u} \vartheta)=-\lambda \nabla p \cdot \nabla \vartheta+b(c) \nu \vartheta \\
& \nabla \cdot(\mathbf{u} \eta)=-\lambda \nabla p \cdot \nabla \eta+b(c) \nu \eta .
\end{aligned}
$$

We see from (3.8) that $p$ is proportional to $\lambda^{-1}$, so that $\lambda \nabla p$ is independent of $\lambda$. Consequently $\nu, \vartheta$, and $\eta$ are independent of $\lambda$, allowing us to set $\lambda=1$.

We impose periodic and other boundary conditions, similar to what was done in [1], to obtain the complete model. The active cell volume fractions satisfy

$$
\begin{aligned}
\partial_{t} \nu(\mathbf{x}, t)-\nabla p \cdot \nabla \nu=-\mu_{v}(c) \nu(\mathbf{x}, t)+ & g\left(s^{*}, c\right) \vartheta\left(\mathbf{x}, s^{*}, t\right) \\
& -h(c) \nu(\mathbf{x}, t)+b(c) \nu(\mathbf{x}, t)(1-\nu(\mathbf{x}, t)),
\end{aligned}
$$

for $x \in B_{t}, t>0$ with conditions

$$
\begin{aligned}
\frac{\partial \nu}{\partial z}=0, & \mathbf{x} \in \Gamma_{B}, t \geq 0 \\
\nu(\mathbf{x}, 0)=\nu_{0}(\mathbf{x}), & \mathbf{x} \in B_{t},
\end{aligned}
$$

where $z$ denotes the spatial variable orthogonal to the surface $\Gamma_{B}$, and $\nu_{0}$ is the initial active cell population. The dormant cell volume fractions satisfy

$$
\begin{aligned}
& \partial_{t} \vartheta(\mathbf{x}, s, t)+\partial_{s}(g(s, c) \vartheta(\mathbf{x}, s, t))-\nabla p \cdot \nabla \vartheta \\
= & -\mu_{v}(s, c) \vartheta(\mathbf{x}, s, t)-b(c) \nu(\mathbf{x}, t) \vartheta(\mathbf{x}, s, t),
\end{aligned}
$$

for $x \in B_{t}, s>s_{0}, t>0$, with conditions

$$
\begin{aligned}
g\left(s_{0}, c\right) \vartheta\left(\mathbf{x}, s_{0}, t\right) & =h(c) \nu(\mathbf{x}, t), & \mathbf{x} \in B_{t}, t>0, \\
\frac{\partial \vartheta}{\partial z} & =0, & \mathbf{x} \in \Gamma_{B}, t \geq 0, s>s_{0}, \\
\vartheta(\mathbf{x}, s, 0) & =0, & \mathbf{x} \in B_{t}, s \geq s_{0} .
\end{aligned}
$$


The fully inert cell volume fractions, including necrotic cells, satisfy

$$
\partial_{t} \eta(\mathbf{x}, t)-\nabla p \cdot \nabla \eta=\mu_{u}(c) \nu(\mathbf{x}, t)+\int_{s_{0}}^{s^{*}} \mu_{v}(s, c) \vartheta(\mathbf{x}, s, t) d s-b(c) \nu(\mathbf{x}, t) \eta(\mathbf{x}, t),
$$

for $x \in B_{t}, t>0$, with conditions

$$
\begin{aligned}
\frac{\partial \eta}{\partial z} & =0, \quad \mathbf{x} \in \Gamma_{B}, t \geq 0, \\
\eta(\mathbf{x}, 0) & =\eta_{0}(\mathbf{x}), \quad \mathbf{x} \in B_{t},
\end{aligned}
$$

where $\eta_{0}$ is the initial inert cell population. Pressure satisfies

$$
\begin{aligned}
-\nabla^{2} p & =b(c) \nu, & \mathbf{x} & \in B_{t}, t \geq 0, \\
p & =0, & \mathbf{x} & \in \Gamma_{t}, t \geq 0, \\
\frac{\partial p}{\partial z} & =0, & \mathbf{x} & \in \Gamma_{B}, t \geq 0 .
\end{aligned}
$$

Let $f=\left[f_{1}, \ldots, f_{m}\right]$ and $c=\left[c_{1}, \ldots, c_{m}\right]$. The chemical species satisfy, for $j=1, \ldots, m$,

$$
\begin{aligned}
-D_{j} \nabla^{2} c_{j} & =f_{j}, \quad \mathbf{x} \in \Omega, t>0, \\
f_{j} & =0, \quad \mathbf{x} \in \Omega \backslash B_{t}, \\
c_{j} & =c_{j}^{*}, \quad \mathbf{x} \in \Gamma_{H_{b}}, t \geq 0, \\
\frac{\partial c_{j}}{\partial z} & =0, \quad \mathbf{x} \in \Gamma_{B}, t \geq 0,
\end{aligned}
$$

where the $D_{j}$ are chemical diffusion coefficients and the $c_{j}^{*}$ are the chemical concentrations in the bulk fluid. The normal velocity of the interface $\Gamma_{B}$ is given by

$$
-\nabla p \cdot \mathbf{n}=-\frac{\partial p}{\partial n}
$$

where $\mathbf{n}$ is the unit outward normal of $\Gamma_{B}$. In practice, to avoid uninhibited growth, some limiting process is added to (3.10r) (see (5.3) below for the case of one spatial dimension).

\section{Computational methodology}

As was done for senescence-structure in $[6,17]$, we often handle a general physiologically structured system such as (2.1a)-(2.1c) more easily if it is transformed to an age-structured system, whether in the statement of the problem, or indirectly in the numerical method [12]. Once our system is transformed to one with just age structure, existing numerical methods become sufficient. However, for dormancy such an approach is ill-advised.

To see this, let $a \geq 0$ represent the time a cell has spent dormant. We make a change of variables so that dormancy, $s(a, t)$, is a separate function of age and time. We then get age-structured equations for $v$,

$$
\begin{aligned}
\partial_{t} v(a, t)+\partial_{a} v(a, t) & =-\tilde{\mu}_{v}(s, c) v(a, t)-D v(a, t), \quad 0<a \leq a^{*}, t>0 \\
v(0, t) & =h(c) u(t), \quad t>0, \\
\partial_{t} s(a, t)+\partial_{a} s(a, t) & =g(s(a, t), c(t)), \quad 0<a \leq a^{*}, t>a \\
s(0, t) & =s_{0}
\end{aligned}
$$


where $\tilde{\mu}_{v}(s, c)=\mu_{v}(s, c)+\partial_{s} g(s, c)$ and $s\left(a^{*}, t\right)=s^{*}$. Setting the initial condition $v_{0}=0$ requires only that we consider $t>a$ for the domain of $s(a, t)$.

For a choice of $g(s, c)=\frac{k_{g} c}{\gamma_{g}+c}$ for scalar $c$, we get

$$
s^{*}=s_{0}+\int_{t-a}^{t} \frac{\gamma_{g}+c(\tau)}{k_{g} c(\tau)} s^{*} d \tau,
$$

so that $a^{*} \rightarrow \infty$ if $c \rightarrow 0$. Since functions with similar behavior to $g$ are natural representations of the dormancy dynamics, we thus find that the original physiologically structured system is more tractable computationally than the equivalent agestructured system for most forms of $g$ that interest us.

To solve directly equations with more general physiological structure, we use an extension of the natural-age-grid Galerkin methods developed for age- and spacestructured systems in $[2,3]$. These methods move the discretization nodes in age smoothly along characteristic lines. The solutions are approximated by piecewise polynomials, rather than moments as was done by de Roos [11]. Our extension of these methods to general physiological structure moves the discretization nodes in the physiological variable along characteristic curves, similar to a method of Sulsky [23], but with the preservation of the property in our methods that each time step need not result in a new discretization node in the physiological variable. This is essential when variation of spatial structure, or any other dynamics in the problem, occurs on a faster time scale than that of the physiological trait. Otherwise, the need to take lots of small time steps would induce many more physiological nodes than are necessary for accuracy, resulting in potentially great loss of efficiency from additional computation or interpolation onto a coarser grid.

To motivate the integration in age and time, we ignore for the moment the discretization in space. We partition the domain $\left[s_{0}, s^{*}\right]$ at each time by the set of nodes $\left\{s_{i}(t)\right\}_{i=0}^{N}$ where $s_{0}(t)=s_{0}$ (in this section $i$ denotes a node in the dormancy discretization rather than species number as above). If $s_{N}(t) \geq s^{*}$, we simply ignore that node and the function value over it until needed. This is not an issue for our choices of $g$. We compute the solution at times $t_{j}$ and let $\Delta t_{j}=t_{j+1}-t_{j}, s_{i, j}=s_{i}\left(t_{j}\right)$, and $\Delta s_{i, j}=s_{i+1, j}-s_{i, j}$. For the last interval we use $\Delta s_{N, j}=\max \left(s^{*}-s_{N, j}, 0\right)$. Although we are not including space in this discussion for reasons of clarity, the presence of spatial structure in a problem will induce different time scales into a problem, making adaptivity and nonuniformity of time intervals an important property of any method used.

For the computations in this paper, we use a piecewise constant approximation space over the domain $\left[s_{0}, s^{*}\right]$. Higher-order approximation spaces can be used, as was done in age in [2]. We define the projection into the space of piecewise constants over the partition of $\left[s_{0}, s^{*}\right]$ by

$$
\Pi\left(v\left(s, t_{j}\right)\right)=\left\{\begin{aligned}
\frac{1}{\Delta s_{i, j}} \int_{s_{i, j}}^{s_{i+1, j}} v\left(s, t_{j}\right) d s, & \text { if } s_{i, j} \leq s<s_{i+1, j}, \\
0, & \text { otherwise. }
\end{aligned}\right.
$$

We make the approximation $V_{i, j} \approx \Pi\left(v\left(s, t_{j}\right)\right)$ via the following algorithm. Let $\Delta s_{\max }$ be the largest we want the first interval in $s$ to be. For most time steps we have $\Delta s_{0, j} \leq \Delta s_{\max }$. In this case we set

$$
s_{i, j+1}=s_{i, j}+\Delta t_{j} g\left(s_{i, j}, c\left(t_{j}\right)\right), \quad \text { for } i=1, \ldots, N .
$$

We choose $\Delta t_{j}$ such that $s_{i, j+1} \geq s^{*}$ for at most one $i$, so as to keep $N$ fixed. 
Let the value $V_{i, j}$ denote the density over $\left[s_{i, j}, s_{i+1, j}\right]$ for $i=1, \ldots, N-1$. We use $\mathcal{B}(t)$ to denote the creation of newly dormant cells at $s_{0}$. Then

$$
\begin{aligned}
V_{i, j+1} & =\frac{\Delta s_{i, j}}{\Delta s_{i, j+1}} V_{i, j}, \quad \text { for } i=1, \ldots, N-1, \\
V_{0, j+1} & =\frac{1}{\Delta s_{0, j+1}}\left(\Delta s_{0, j} V_{0, j}+\Delta t_{j} \mathcal{B}\left(t_{j}\right)\right) \\
V_{N, j+1} & =\frac{\Delta s_{N-1, j}-\Delta t_{j}}{\Delta s_{N, j+1}} V_{N-1, j} .
\end{aligned}
$$

Because the applications in this paper provide for the first extension of the methods presented in $[2,3]$, we have kept $\mathcal{B}(t)$ general in this part of the presentation of the method. In our case we have $\mathcal{B}\left(t_{j}\right)=g\left(s_{0}, c(t)\right) v\left(s_{0}, t\right)$. Also, if $g$ is independent of $s$, we have $\Delta s_{i, j} / \Delta s_{i, j+1}=1$ for $i=1, \ldots, N-1$.

If $\Delta s_{0, j}>\Delta s_{\max }$, we introduce a new node and set

$$
\begin{aligned}
s_{i+1, j+1}=s_{i, j}+\Delta t_{j} g\left(s_{i, j}, c\left(t_{j}\right)\right), & \text { for } i=1, \ldots, N-1, \\
V_{i+1, j+1}=\frac{\Delta s_{i, j}}{\Delta s_{i, j+1}} V_{i, j}, & \text { for } i=0, \ldots, N-1,
\end{aligned}
$$

for the intermediate intervals, and set

$$
\begin{aligned}
s_{1, j+1} & =\Delta t_{j} g\left(s_{0}, c\left(t_{j}\right)\right), \\
V_{0, j+1} & =\frac{\Delta t_{j} \mathcal{B}(t)}{\Delta s_{0, j+1}}, \\
V_{N, j+1} & =\frac{\Delta s_{N-1, j} V_{N-1, j}+\left(\Delta s_{N, j}-\Delta t_{j} g\left(s^{*}, c\left(t_{j}\right)\right)\right) V_{N, j}}{\Delta s_{N, j+1}},
\end{aligned}
$$

for the first and last intervals.

The above calculations account for transport in the physiological variable, entry into dormancy, and exit from dormancy. Upwind differences approximate the advection terms in space. Center differences approximate the diffusion terms in space. Backward Euler formulæ, embedded in step-doubling with local extrapolation, approximate the time derivatives. We choose step-doubling because it is easy to implement and creates a likely second-order correct time integration scheme [4]. This notion of convergence order is a theoretical measure; because step-doubling is an adaptive method, we use a tolerance parameter for local truncation error rather than refine a fixed time step. We note that other suitable choices of time-integration scheme certainly exist and our choice is not essential to our computational results or the broader relevance of our discretization of the physiological variable.

\section{Computations}

In this section we present computational results for models of batch cultures, chemostat cultures, and biofilms. We have two goals for these examples: 1) to illustrate our modeling and simulation approach, and 2) show that even under very simple assumptions biofilm and planktonic cultures can differ substantially.

We make several simplifying assumptions in order to identify core differences between the behavior in biofilm and planktonic cultures. In particular, we assume no mortality for dormant cells (biologically reasonable on time scales considered here) and substrate-independent mortality for active cells (to isolate other advantages to 
dormancy). We also do not consider the case of dormancy as a defense against antimicrobial attack.

We use the same parameters for planktonic and biofilm cultures to emphasize the effects of spatial structure. Because the parameters are taken from the biofilm literature, we can interpret the planktonic cases as being suspended activated flocs.

5.1. Batch culture dormancy as a response to nutrient deprivation. We let $c(t)$ be a scalar value representing nutrient. For a two-species model $(i=2$ in system (2.1)), we choose the functional forms

$$
\begin{aligned}
b_{i}(c) & =\frac{k c}{\gamma+c}, \\
h_{i}(c) & =\frac{k_{h}}{\zeta+c}+\epsilon_{h}, \\
g_{i}(s, c) & =\max \left(\frac{k_{g_{i}}(c-\hat{c})}{\gamma+(c-\hat{c})}, 0\right), \\
\mu_{u_{i}}(c) & =\mu_{u}, \\
\mu_{v_{i}}(s, c) & =0, \\
v_{0}(s) & =1, \\
u_{0} & =0,
\end{aligned}
$$

with constant $\mu_{u}$, rate constants $k, k_{h}, k_{g}$, saturation constant $\gamma$, Monod constant $\zeta$, critical substrate $\hat{c}$. We highlight that the two species differ only in the selection of $k_{g_{i}}$.

We will examine two types of substrate usage and introduce the yield constant $Y$. The first is no usage of substrate by dormant cells,

$$
f=f_{1}(c, u, v)=-\frac{k c}{Y(\gamma+c)}\left(u_{1}+u_{2}\right),
$$

and the second is some usage, increasing with $s$, for cells to transit and exit dormancy,

$$
f=f_{2}(c, u, v)=-\frac{k c}{Y(\gamma+c)}\left(u_{1}+u_{2}+\int_{0}^{1} e^{-(1-s) / k_{g}}\left(v_{1}+v_{2}\right) d s\right) .
$$

The dependence of the integral weight in $f_{2}$ on $k_{g}$ reflects the assumption that two cells which transit dormancy under identical substrate levels will consume an equal amount of total substrate, even though their transit times may differ. In other words, the amount of resources to transit and exit dormancy does not differ appreciably for our two cells under similar conditions, just the length of time to do so.

We take the baseline parameters $k=1 / 4 \mathrm{hr}, \gamma=4 \mathrm{~g}_{\mathrm{CODB}} / \mathrm{m}^{3}, \epsilon_{h}=0.05, \mu_{u}=$ $0.005 / \mathrm{hr}$, and $Y=0.63 \mathrm{~g}_{\mathrm{CODB}} / \mathrm{g}_{\mathrm{CODS}}[24]$. The units $\mathrm{g}_{\mathrm{CODB}}$ and $\mathrm{g}_{\mathrm{CODS}}$ are the chemical oxygen demand of biomass and substrate mass, respectively. We assume a small $\zeta$, say $\zeta=\gamma / 20=\mathrm{g}_{\mathrm{CODB}} / 5 \mathrm{~m}^{3}$. Given the linkage between the nutrient needed to reproduce and that needed to transit dormancy, we set $\hat{c}$ to be proportional to $\zeta$. For definiteness and to halt transit through dormancy at minimum substrate, we use $\hat{c}=2 \zeta$. We require $k_{h} / \zeta \geq 1 / 24 \mathrm{hr}$, so take $k_{h}=\mathrm{g}_{\mathrm{CODB}} /\left(6 \mathrm{hr} \mathrm{m}^{3}\right)$.

We set the dormancy domain to be $s_{0}=0$ and $s^{*}=1$, and assume $24 \mathrm{hr}$ emergence for species 1 when $c-\hat{c}=\gamma$ (ample substrate), so that $k_{g_{1}}=1 / 12 \mathrm{hr}$. To compare the relatively fast dormancy time of a normal planktonic species with one that reawakens more slowly, we set $k_{g_{2}}=1 / 24 \mathrm{hr}$ for species 2 . 


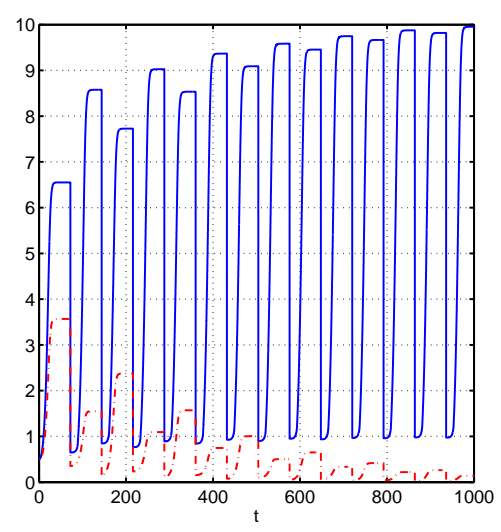

(a) Total Live-Cell Density

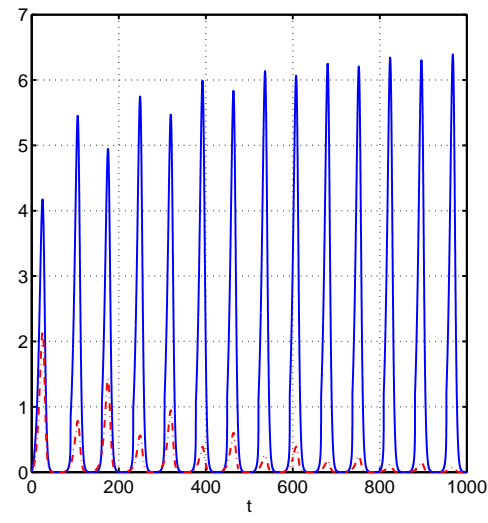

(b) Active-Cell Density

FIG. 5.1. Results for the batch model with $f=f_{2}$ for two species with $k_{g_{1}}=1 / 12$ (solid/blue) and $k_{g_{2}}=1 / 24$ (dashed/red) showing (a) total live-cell density and (b) active-cell density. Each subpopulation was recultured onto new media every 72 hours in proportion to their densities, summing to unity. Functional forms are as in (5.1) with parameters $k=1 / 4 h r, \gamma=4 g_{C O D B} / m^{3}, \epsilon_{h}=0.05$, $\mu_{u}=0.005 / h r, Y=0.63 g_{C O D B} / g_{C O D S}, \zeta=g_{C O D B} / 5 m^{3}, \hat{c}=2 \zeta$, and $k_{h}=g_{C O D B} /\left(6 h r m^{3}\right)$. The dormancy domain is $[0,1]$. Time is measured in hours. In the long run, the slow waker is driven to extinction in both cases, $f=f_{1}$ and $f=f_{2}$. The two cases do not differ qualitatively and only have minor quantitative differences.

We conducted simulations with two species where each subpopulation was recultured into fresh media every $4,8,12,24,48,72,168$, and 240 hours in proportion to their densities, summing to unity. In all cases the fast-waker population $\left(k_{g_{1}}=1 / 12\right.$ $\mathrm{hr}$ ) outgrew the slow-waker population $\left(k_{g_{2}}=1 / 24 \mathrm{hr}\right)$. This outcome is not surprising; conditions favor microbes that more rapidly resuscitate. Results for 72-hour reculturing are shown in Figure 5.1. We characterize the decay process of the slow waker population as time $\left(t_{d}\right)$ and number of reculturing events $\left(n_{r}\right)$ until the slow waker population is limited to $10 \%$ of the total biomass at the time of the reculturing event. The results for our range of reculturing interval times $\left(t_{c}\right)$ are

\begin{tabular}{|c||c|c|c|c|c|c|c|c|}
\hline$t_{c}$ & 4 & 8 & 12 & 24 & 48 & 72 & 168 & 240 \\
\hline$t_{d}$ & 1476 & 1368 & 1164 & 384 & 288 & 432 & 1008 & 1200 \\
\hline$n_{r}$ & 369 & 171 & 97 & 16 & 6 & 6 & 6 & 5 \\
\hline
\end{tabular}

5.2. Chemostat culture dormancy as a response to nutrient deprivation. We use the functional forms and parameter values of Section 5.1, with $d_{0}=k / 2$. The active population, dormant population, and nutrient relax to a periodic oscillation. We study periodic oscillations in substrate level in imitation of the periodic recultured batch system. Note (and see later analysis) that dormancy would seem unlikely to be of benefit in a steady chemostat. Possible advantage, or not, of dormancy in a periodic chemostat system is less evident, especially if the oscillation and dormancy times scales are comparable.

For two competing species, the only difference being $k_{g_{1}}=1 / 12$ vs. $k_{g_{2}}=1 / 24$, the results are shown in Figure 5.2. As in the batch case, the faster waker outcompetes the slower waker in the long run. This is true for a wide range of periods, $C(t)=$ $8+8 \cos (2 \pi t / \varpi)$ with $\varpi=0.5,1,4,8,12,24,48,72,168$, and 240 hours, verifying 


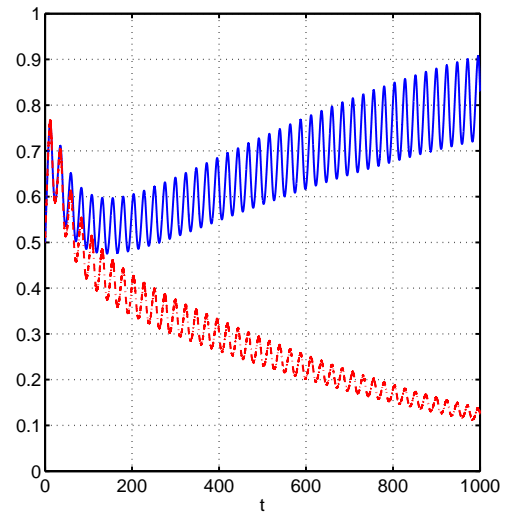

(a) Total Live-Cell Density

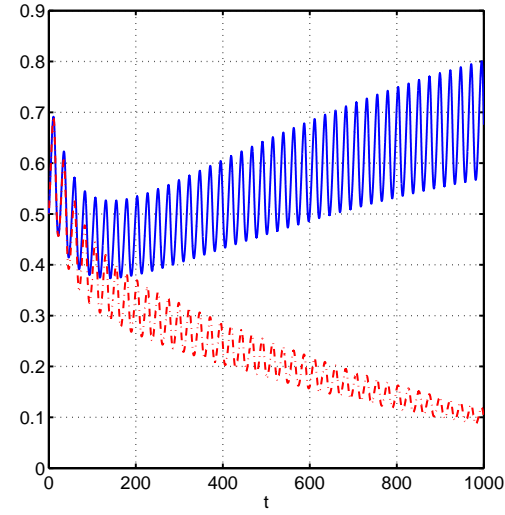

(b) Active-Cell Density

FIG. 5.2. Results for the chemostat model with $f=f_{2}$ for two species with $k_{g_{1}}=1 / 12($ solid $/$ blue line) and $k_{g_{2}}=1 / 24$ (dashed/red line) showing (a) total live-cell density and (b) active-cell density. We take $d_{0}=k / 2$ and $C(t)=8+8 \cos (\pi t / 12)$, i.e. $\varpi=24$. All other parameters are as in Figure 5.1. In the long run, the slow waker is driven to extinction in both cases, $f=f_{1}$ and $f=f_{2}$, and the cases do not differ qualitatively and only have minor quantitative differences.

the asymptotics predictions for short and long periods and extending to intermediate periods (see Section 7.2).

As expected, the fast waker also outcompetes the slow waker in the case of a steady, rather than oscillating, nutrient source (as predicted in Section 7.1).

5.3. Biofilm dormancy as a response to nutrient deprivation. We present computational results for one spatial dimension (height of the biofilm), and explicit dormancy structure for the dimensional system (3.10). The height of the biofilm, $\Gamma_{B}$, is regulated using an erosion or shedding term at the biofilm/substrate interface (a standard device in biofilm models; see e.g. [14]). We modify (3.10r) in one spatial dimension to obtain

$$
\frac{\partial \Gamma_{B}}{\partial t}=-\left.\frac{\partial p}{\partial z}\right|_{z=\Gamma_{B}}-\alpha \Gamma_{B}^{2}
$$

where $\alpha$ is the erosion or shedding coefficient.

In the biofilm model, the two choices of substrate usage (5.2) become

$$
\begin{aligned}
& f=f_{1}(c, \nu, \vartheta)=-\frac{k \rho^{*} c}{Y(\gamma+c)} \nu, \\
& f=f_{2}(c, \nu, \vartheta)=-\frac{k \rho^{*} c}{Y(\gamma+c)}\left(\nu+\int_{0}^{1} e^{-(1-s) / k_{g}} \vartheta d s\right) .
\end{aligned}
$$

Otherwise we use the functional forms and parameter values of Section 5.1, with the addition of $\rho^{*}=10^{4} \mathrm{~g}_{\mathrm{CODB}} / \mathrm{m}^{3}$ and $D=10^{-4} \mathrm{~m}^{2} /$ day [24]. Initial conditions for all runs are $\nu=1$ up to a height of $50 \mu \mathrm{m}$.

Our computational results suggest that there is no disadvantage for slow reawakening when $f_{1}$ is used for substrate usage - the amount of live active biomass at each 


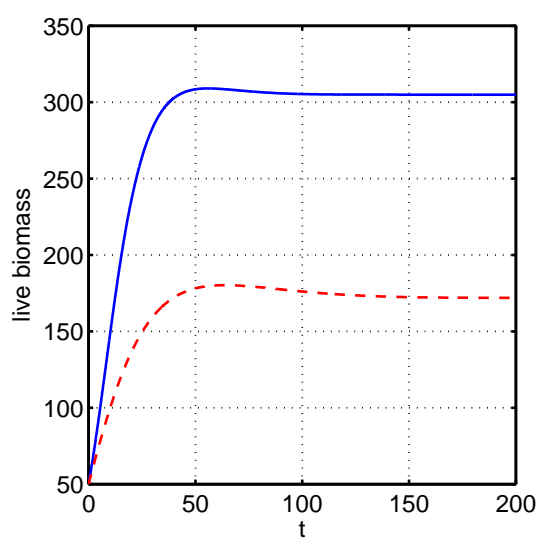

(a) Live Biomass

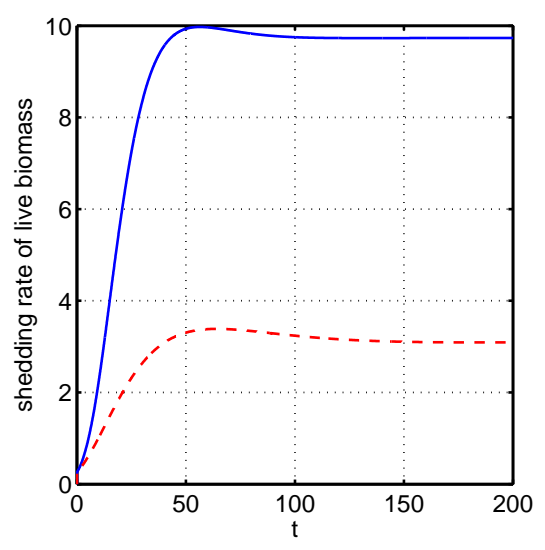

(b) Shedding Rate of Live Biomass

FIG. 5.3. Results for the biofilm model, system (3.10) using $f_{1}$ given by (5.4a). We consider two cases of background substrate, $c^{*}=10$ (normal, solid/blue line) and $c^{*}=5$ (dashed/red line). Functional forms are as in (5.1). Total live biomass and shedding rate of live biomass is independent of $k_{g}$ for all $t$. Computations shown are for $k_{g}=1 / 12$, which are identical to those conducted for $k_{g}=1 / 24, k_{g}=1 / 4$, and $k_{g}=1$. Other parameters are $k=1 / 4 h r, \gamma=4 g_{C O D B} / m^{3}, \epsilon_{h}=0.05$, $\mu_{u}=0.005 / h r, Y=0.63 g_{C O D B} / g_{C O D S}, \zeta=g_{C O D B} / 5 m^{3}, k_{h}=g_{C O D B} /\left(6 h r m^{3}\right)$, and $\hat{c}=2 \zeta$. The dormancy domain is $[0,1]$. Time is measured in hours. Biomass is measured as height in microns times volume fraction.

height level is the same, as is the amount of fully inert (i.e. dead) cells. Only the distribution of dormant cells differs. Figure 5.3 shows the live biomass and shedding rate of live biomass for two background substrate levels (normal $c^{*}=10$ and low $c^{*}=5$ ), which is independent of $k_{g}$.

In retrospect, this might be expected. The combined population of cells consuming substrate is dictated by the substrate profile. In the case of only active cells consuming substrate, the population of active cells is fixed by the substrate, independent of the reawakening process (represented by $k_{g}$ ). Since only active cells can die, the dead cell profile is also independent of $k_{g}$. We note that our two choices of the transit rate parameter $k_{g}$ are significantly slower than the cell division time parameter $k$. To assure ourselves that this insensitivity to $k_{g}$ was not due to dominance of the effects of $k_{g}$ by $k$, we examined additional cases of even faster wakers $\left(k_{g}=k=1 / 4\right.$ and $k_{g}=1$ ). The insensitivity of the live biomass and shedded live biomass rate held. In the case where dormant cells use substrate to transit dormancy and reawaken $\left(f_{2}\right)$, the advantage goes to the slower wakers - in contrast to the results from our planktonic simulations. This can be understood by again considering that the substrate profile dictates the population values concerned. Faster waker populations have, in their steady state dormancy distributions (see Figure 5.4), more of their cells nearer to reawakening, and hence using more substrate. This substrate is in effect being taken away from contributing to active-cell population growth, which is what drives the total growth of the biofilm.

This effect is not monotone, however. Although for the most biologically relevant choices (small $k_{g}$ ) this holds, and the choice of $k_{g}=1 / 24$ yields more live biomass than faster reawakening (and $k_{g}=1 / 48$ even more so), we find that for values of $k_{g}>k$ (i.e., dormancy transit time relatively short in comparison to growth time) we begin to see 

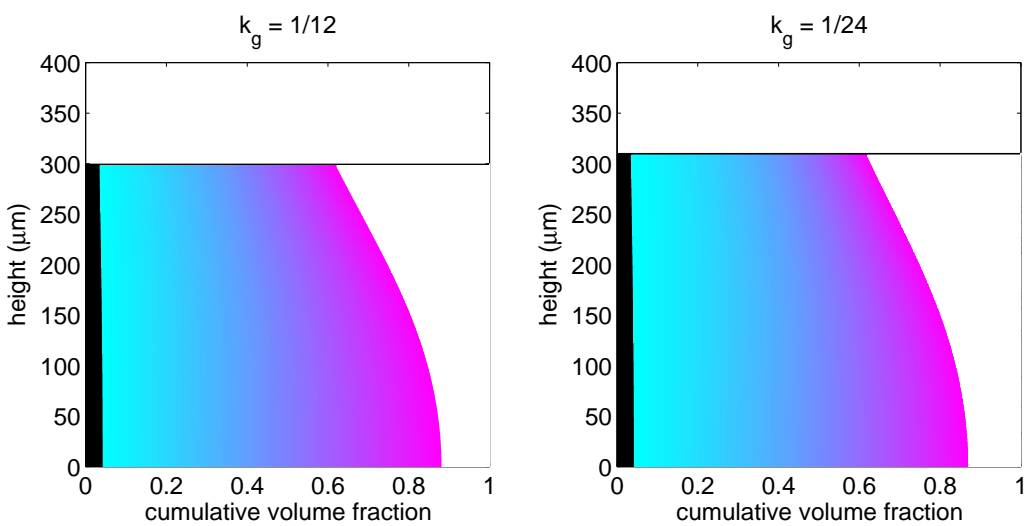

FIG. 5.4. Results for the biofilm model, system (3.10) using $f_{2}$ given by (5.4b). Functional forms are as in (5.1). Parameters are as in Figure 5.3, with the exception that $c^{*}$ is fixed at 10. The two figures differ in the values of $k_{g}$. Time is measured in hours. The horizontal width of a color constitutes the volume fraction of cells in the corresponding state. White denotes active cells, black denotes fully inert cells, and the spectrum from cyan or light grey to magenta or dark grey denotes dormancy from 0 to 1 . In contrast to the results of the equivalent batch and chemostat computations, slow reawakening has an advantage over fast reawakening in the case of using $f_{2}$ for substrate usage.

improved live biomass yield. The steady-state values of live biomass and live biomass shedding rate are

\begin{tabular}{|r||c|c|c|c|c|c|c|}
\hline$k_{g}$ & $1 / 48$ & $1 / 24$ & $1 / 12$ & $1 / 4$ & $1 / 2$ & 1 & 2 \\
\hline live biomass & 301.6 & 295.3 & 285.0 & 279.0 & 281.7 & 285.3 & 287.0 \\
\hline shedding rate & 9.526 & 9.133 & 8.511 & 8.155 & 8.317 & 8.525 & 8.628 \\
\hline
\end{tabular}

To understand this nonmonotonicity of live biomass and shedding rate as a function of $k_{g}$, we can interpret the dormant cell population as a second population that consumes resources (related to $v(x, \cdot, t)$ and ultimately $g(\cdot, c)$ ) and is both a source for live biomass (related to $g\left(s^{*}, c\right)$ ) and a sink (related to $g\left(s_{0}, c\right)$, which is independent of $\left.k_{g}\right)$. When $g\left(s^{*}, c\right)$ is larger, it seems that the tradeoff between substrate usage and this net yield (source minus sink) begins to change. We note that $k_{g}=2$ is a large velocity term for a domain of $[0,1]$.

In cases where $c^{*}$ is a periodic function of time similar to the periodic chemostat model,

$$
c^{*}(t)=8+8 \cos (2 \pi t / \varpi)
$$

with $\varpi=1,8,24,48,72,168$, and 240 hours, we found similar results for both choices of $f$, namely that there was no advantage to either strategy when $f=f_{1}$, and there was an advantage to slow reawakening when $f=f_{2}$. 


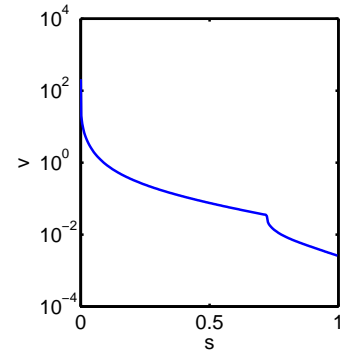

(a) $v_{1}$

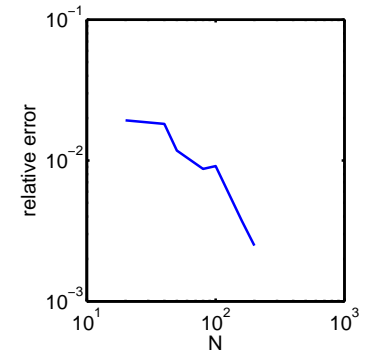

(b) Error in $\int_{0}^{1} v d s$

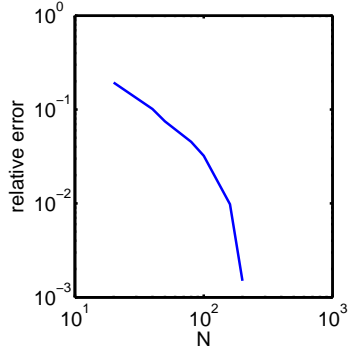

(c) Error in $v_{1}$

FIG. 6.1. Convergence of the method in s.

\section{Convergence study of the numerical methods}

In this section we examine the convergence of the discretization of the physiological variable, $s$, presented in Section 4. We use as an example the batch computations in Section 5.1 with $f=f_{2}$. The choice of $f_{2}$ better illustrates the relevance of the method to other problems. We used a reculturing interval time of $t_{c}=72$ out to five reculturing periods $(t=360)$.

To measure convergence, we need to take into consideration the nature of the solution. When substrate density is very low, cells enter dormancy with $s=0$ and remain there, forming a singularity in the true solution. Any numerical discretization of this solution constitutes a regularization or mollification of a delta function, as illustrated in Figure 6.1(a). Rather than trying to measure accuracy as convergence to a singularity, in this context we are interested in conserving total biomass. We thus study convergence in total biomass, $\int_{0}^{1} v_{i} d s$, the bulk of which is near $s=0$. However, because of the form of $f_{2}$ (which depends on $s$ in a manner that is more heavily weighted towards $s=1$ ) and the importance in accurately measuring cell populations exiting from dormancy, the convergence of $v_{i}$ away from $s=0$ is also of interest.

We note that the way in which the natural-grid methods handle the singularity at $s=0$ is one more reason to use this approach.

We study convergence by increasing the number of intervals, $N$. Recall that the interval widths vary and are determined by the characteristics of the problem. Hence there is no simple parameter called $\Delta s$ to reduce. We compute solutions with $N=20,40,50,80,100,160,200$ and compare them to a calculation with $N=300$ intervals by interpolating onto a common grid. Time is integrated using step-doubling extrapolation. As mentioned in Section 4, there are other suitable time integration methods. Rather than specifying a fixed $\Delta t$, step-doubling determines a suitable $\Delta t$ for each step in time given a tolerance parameter for local truncation error. We require the relative local truncation error in the 2-norm to be measured as less than 0.001 .

Figure 6.1(b) indicates superlinear asymptotic convergence of the relative error in the 2-norm in computing total biomass, $\int_{0}^{1} v_{1} d s$. The slope in the log-log plane is roughly -1.8 for the higher values of $N$, which corresponds to a convergence order of roughly 1.8. A theoretical limit is provided by analyses for natural-grid methods for age-structured systems, methods that constitute special cases of the method presented in this paper $[2,3]$. For the case where the solution is approximated by piecewise constant functions, we can obtain second-order convergence in the age variable. One 
additional source of error when going from age to a more general physiological variable such as dormancy is that $g$ needs to be approximated (for age structure $g=1$ ).

Figure 6.1(c) indicates superlinear asymptotic convergence of the relative error in the 2 -norm in $v_{1}$ on the interval $[0.1,1]$.

The convergence study presented in this section suggests the value of an energy analysis of the methods similar to those in $[2,3,5]$. An additional consideration is the effect of the error in computing $g$ on the estimates. This analysis is primarily suitable for a specialized numerical analysis audience, and most appropriately done for the first time in a more general context of physiological structure rather than a rather specific biofilm model. The work presented in this paper helps to illustrate the broader relevance of a more theoretical work in numerical analysis.

\section{Long-time behavior}

In this section we examine the long-time behavior of chemostat models for steady and periodic cases.

7.1. Steady chemostat. We consider the long-time behavior of the steady $\left(C_{0}(t)=C_{0}\right)$ chemostat system by studying the time-independent solution of Equation (2.1) with $i=1$. In the steady state $c(t)=c$, so that we can define a new dormancy coordinate $a \in\left[0, a^{*}\right]$ by

$$
a(s)=\int_{s_{0}}^{s} \frac{d s^{\prime}}{g\left(s^{\prime}, c\right)},
$$

with $a^{*}=a\left(s^{*}\right)$. Setting the time derivative to zero, (2.1b) together with $(2.1 \mathrm{c})$ can be solved to obtain

$$
v(a)=\frac{h(c) u}{g\left(a^{*}, c\right)} e^{-d_{0} a} e^{-\int_{0}^{a} \mu_{v}\left(a^{\prime}, c\right) d a^{\prime}},
$$

where $u$ is the steady state value of the active cell density and $s$ has been replaced by $a$. The first exponential factor accounts for loss due to washout and the second for loss due to death. Plugging into (2.1a) and again setting the time-derivative to zero, we obtain

$$
0=u\left[b(c)-\mu_{u}(c)-d_{0}-\left(1-e^{-d_{0} a^{*}} e^{-\int_{0}^{a^{*}} \mu_{v}(a, c) d a}\right) h(c)\right] .
$$

If $u \neq 0$, then the second factor of (7.3) provides an equation for $c$. Assuming that $\mu_{u}, \mu_{v}$, and $h$ are all decreasing functions of $c$, we can write that second factor in the form $b(c)-\bar{\alpha}-\tilde{\alpha}(c)$, where $\bar{\alpha}$ is a constant and $\tilde{\alpha}(c)$ is a decreasing function of $c$ such that $\tilde{\alpha}(c) \rightarrow 0$ as $c \rightarrow \infty$. Thus, assuming that $b(c)$ is a monotone increasing function of $c$ and that $b(c)>\bar{\alpha}$ for $c$ sufficiently large, then it follows that there is a unique value of $c$ that solves (7.3) with $u \neq 0$. However if that value is larger than $C_{0}$ or if $b(c)<\bar{\alpha}$ for all $c$, then the only admissible solution of (7.3) is $u=0$ (washout).

Finally, given the solution for $c$, Equation (2.1d) can be solved to obtain the long-time behavior of $u$ by setting the time derivative to zero ${ }^{1}$. In the case that $f$ is monotone increasing in its arguments, we obtain a unique solution for $u$.

For two (or more) species competing in the same chemostat, the one that has the steady-state solution with smallest value of substrate $c$ is the only long-time survivor

\footnotetext{
${ }^{1}$ If $u=0$, then $(2.1 \mathrm{~d})$ requires that $d_{0}\left(C_{0}-c\right)=f(c, 0,0)$; generally $f(c, 0,0)=0$, in which case $c=C_{0}$.
} 
[21]; it excludes the other species by continually reducing substrate until the substrate level is below the others' steady-state requirements. Thus, from (7.3) it is apparent that the smaller the size of $h$ (i.e., the lesser the likelihood of entry to dormancy) and the smaller the value of $a^{*}$ (i.e., the shorter the dormancy period), the more competitive the species. Note that a species which does not go dormant at all will outcompete an otherwise similar species which does.

\subsection{Periodic chemostat}

We consider next the long-time behavior of a periodic chemostat with input substrate concentration $C_{0}(\omega t)$ where $C_{0}(a+1)=C_{0}(a)$, and offer asymptotics for two special cases (again with $i=1$ ).

7.2.1. Fast Oscillations. We suppose that the chemostat oscillation period is short compared to all other time scales of interest. In this limit, the chemostat will oscillate many times before the microbial inhabitants can react. This intuition suggests a multiple time scale expansion with a slow time $t_{1}$ and a fast time $t_{2}$ defined by

$$
t_{1}=t, \quad t_{2}=\epsilon^{-1} t
$$

where $\epsilon=\omega^{-1} \ll \tau$ for any inherent time scale $\tau$ in the system. Note $C_{0}=C_{0}\left(t_{2}\right)$. We expand

$$
\begin{aligned}
& u=u_{0}\left(t_{1}, t_{2}\right)+\epsilon u_{1}\left(t_{1}, t_{2}\right)+\ldots, \\
& v=v_{0}\left(s, t_{1}, t_{2}\right)+\epsilon v_{1}\left(s, t_{1}, t_{2}\right)+\ldots, \\
& c=c_{0}\left(t_{1}, t_{2}\right)+\epsilon c_{1}\left(t_{1}, t_{2}\right)+\ldots,
\end{aligned}
$$

We suppose that the solution $(u, v, c)$ approaches periodicity with period $\omega^{-1}$ for long times, so we look for a solution independent of slow time $t_{1}$, i.e.,

$$
\begin{aligned}
& u=u_{0}\left(t_{2}\right)+\epsilon u_{1}\left(t_{2}\right)+\ldots, \\
& v=v_{0}\left(s, t_{2}\right)+\epsilon v_{1}\left(s, t_{2}\right)+\ldots, \\
& c=c_{0}\left(t_{2}\right)+\epsilon c_{1}\left(t_{2}\right)+\ldots,
\end{aligned}
$$

In this case, $d / d t=\epsilon^{-1} d / d t_{2}$. Then to lowest order $\left(=O\left(\epsilon^{-1}\right)\right)$, system (2.1) becomes

$$
\frac{\partial u_{0}}{\partial t_{2}}=\frac{\partial v_{0}}{\partial t_{2}}=\frac{\partial c_{0}}{\partial t_{2}}=0
$$

so that $u_{0}, c_{0}$ are constants and $v_{0}=v_{0}(s)$, i.e.,

$$
\begin{aligned}
& u=u_{0}+\epsilon u_{1}\left(t_{2}\right)+\ldots, \\
& v=v_{0}(s)+\epsilon v_{1}\left(s, t_{2}\right)+\ldots, \\
& c=c_{0}+\epsilon c_{1}\left(t_{2}\right)+\ldots,
\end{aligned}
$$

At the next order $\left(=O\left(\epsilon^{0}\right)\right)$, system $(2.1)$ becomes

$$
\begin{aligned}
\frac{d}{d t_{2}} u_{1}\left(t_{2}\right) & =b\left(c_{0}\right) u_{0}-\mu_{u}\left(c_{0}\right) u_{0}+g\left(s^{*}, c_{0}\right) v_{0}\left(s^{*}\right)-h\left(c_{0}\right) u_{0}-d_{0} u_{0}, \\
\frac{\partial}{\partial t_{2}} v_{1}\left(s, t_{2}\right) & =-\partial_{s}\left(g\left(s, c_{0}\right) v_{0}(s)\right)-\mu_{v}\left(s, c_{0}\right) v_{0}(s)-d_{0} v_{0}(s), \\
\frac{\partial}{\partial t_{2}} c_{1}\left(t_{2}\right) & =-f\left(c_{0}, u_{0}, v_{0}(\cdot)\right)+d_{0}\left(C_{0}\left(t_{2}\right)-c_{0}\right),
\end{aligned}
$$


with $g\left(s_{0}, c_{0}\right) v\left(s_{0}\right)=h\left(c_{0}\right) u_{0}$. Averaging over a period $\omega^{-1}$, we obtain

$$
\begin{aligned}
& 0=b\left(c_{0}\right) u_{0}-\mu_{u}\left(c_{0}\right) u_{0}+g\left(s^{*}, c_{0}\right) v_{0}\left(s^{*}\right)-h\left(c_{0}\right) u_{0}-d_{0} u_{0} \\
& 0=-\partial_{s}\left(g\left(s, c_{0}\right) v_{0}(s)\right)-\mu_{v}\left(s, c_{0}\right) v_{0}(s)-d_{0} v_{0}(s), \\
& 0=-f\left(c_{0}, u_{0}, v_{0}(\cdot)\right)+d_{0}\left(\bar{C}_{0}-c_{0}\right)
\end{aligned}
$$

with $g\left(s_{0}, c_{0}\right) v\left(s_{0}\right)=h\left(c_{0}\right) u_{0}$, where $\bar{C}_{0}$ is the average of $C_{0}\left(t_{2}\right)$ over one chemostat oscillation period. This system, the same as was solved previously in the steady chemostat case except with $\bar{C}_{0}$ replacing $C_{0}$, has essentially the same solutions for $u_{0}, v_{0}(s)$, and $c_{0}$; the next order terms $u_{1}\left(t_{2}\right), v_{1}\left(s, t_{2}\right), c_{1}\left(t_{2}\right)$ add a correction of $O(\epsilon)$. Note thus that the same conclusion holds: a species without dormancy will outcompete an otherwise similar species which can go dormant (because the fast oscillating chemostat acts like a steady chemostat with input substrate $\bar{C}_{0}$ ).

7.2.2. Slow oscillations. We suppose now that the chemostat oscillation period is long compared to all other time scales of interest, i.e., that the chemostat can nearly reach equilibrium before input $C_{0}(\omega t)$ changes noticeably. Intuition again suggests a multiple time scale expansion with a slow time $t_{1}$ and a fast time $t_{2}$, in this case defined by

$$
t_{1}=\epsilon t, \quad t_{2}=t,
$$

where $\epsilon=\omega \ll \tau^{-1}$ for any inherent time scale $\tau$ in the system. Note that $C_{0}=C_{0}\left(t_{1}\right)$. We expand

$$
\begin{aligned}
& u=u_{0}\left(t_{1}, t_{2}\right)+\epsilon u_{1}\left(t_{1}, t_{2}\right)+\ldots, \\
& v=v_{0}\left(s, t_{1}, t_{2}\right)+\epsilon v_{1}\left(s, t_{1}, t_{2}\right)+\ldots, \\
& c=c_{0}\left(t_{1}, t_{2}\right)+\epsilon c_{1}\left(t_{1}, t_{2}\right)+\ldots,
\end{aligned}
$$

We suppose quasi-equilibrium in the sense that $u_{0}, v_{0}$, and $c_{0}$ are independent of fast time $t_{2}$. Noting that $d / d t=\epsilon \partial / \partial t_{1}+\partial / \partial t_{2}$, then at its slowest, $\epsilon^{0}$ order, system (2.1) becomes

$$
\begin{aligned}
& 0=b\left(c_{0}\right) u_{0}-\mu_{u}\left(c_{0}\right) u_{0}+g\left(s^{*}, c_{0}\right) v_{0}\left(s^{*}\right)-h\left(c_{0}\right) u_{0}-d_{0} u_{0}, \\
& 0=-\partial_{s}\left(g\left(s, c_{0}\right) v_{0}\right)-\mu_{v}\left(s, c_{0}\right) v_{0}-d_{0} v_{0} \\
& 0=-f\left(c_{0}, u_{0}, v_{0}(\cdot)\right)+d_{0}\left(C_{0}\left(t_{1}\right)-c_{0}\right),
\end{aligned}
$$

with $g\left(s_{0}, c_{0}\right) v\left(s_{0}\right)=h\left(c_{0}\right) u_{0}$. Note that $t_{1}$ is essentially a parameter appearing explicitly only in the input substrate concentration $C_{0}\left(t_{1}\right)$. Thus to zeroth order, the quantities $u, v$, and $c$ obey system (7.15), which is the same as the steady chemostat except with parameterized input substrate. Hence we again conclude that a species that does not go dormant will outcompete an otherwise similar one that does. Note one caveat though: if $C\left(t_{1}\right)$ drops below the minimum required to sustain a particular population (see Section 7.1) at any point in its cycle, then extinction may occur.

7.2.3. Computational validation of asymptotics. Recall that for a wide range of periods, $C(t)=8+8 \cos (2 \pi t / \varpi)$ with $\varpi=0.5,1,4,8,12,24,48,72,168$, and 240 hours, we have verified the asymptotics predictions for short and long periods, and extending these to intermediate periods.

Computations of competing species in a chemostat, where one species undergoes no dormancy $(h=0)$ and the other undergoes dormancy with parameter $k_{g}=1 / 12$, 
confirm the results of Section 7.2.1 (using $C(t)=8+8 \cos (4 \pi t)$ where $t$ is measured in hours) and Section 7.2.2 (using $C(t)=8+8 \cos (\pi t / 12)$ where $t$ is measured in hours). A species without dormancy capability will outcompete an otherwise similar species which can go dormant, under both fast and slow oscillations in nutrient.

\section{Conclusions}

Our modeling and simulation results show either no disadvantage or some advantage for slow reawakening cells in biofilms - under our simplifying assumption of substrate-independent mortality and the two cases of substrate usage presented. In contrast, dormancy-capable cells in well-mixed, planktonic systems (e.g. batch and chemostat cultures) appear to have less advantage over "regular" cells. In the absence of spatially structured populations, live biomass is maximized by the fastest possible exit from dormancy.

Note though that we present here a somewhat awkward comparison between the planktonic and biofilm systems as a consequence of the absence of direct competition within our biofilm model. Modeling methodology for multispecies competition in biofilms is still, in our view, unsettled [15] and hence we prefer not to address the issue here. Multispecies competition within a biofilm should more appropriately be resolved outside the context of physiological structure. Nevertheless, we feel we have been able to argue that the behavior in biofilm is not simply congruent to that in planktonic cultures, and have identified a potential explanation for the observation that dormancy times are longer in biofilms than planktonic cultures.

We make no claims as to the definitiveness of these results in identifying the mechanisms underlying the observed differences in reawakening in biofilm and planktonic cultures, but rather use them to show that it is natural to look to the spatial structure of biofilm and the physiological structure of both types of systems to understand why reawakening from dormancy can differ in these two states. As most lab populations are of the well-mixed batch or chemostat sort, and many natural populations are of the spatially-structured biofilm sort, this presents a cautionary warning for the use of typical laboratory systems for the characterization of natural ones.

We remark that we have only considered here dormancy response in the context of resource deprivation. Dormancy is also likely an effective defense strategy against antimicrobial agents - many antimicrobials are only effective against metabolically active targets. Thus the presence of antimicrobials reinforces the utility of dormancy in biofilms and also may advantage dormancy-capable populations in well-mixed cultures. The nature of dormancy as defense could itself benefit from modeling studies.

More generally, beyond dormancy specifically, recent studies suggest that phenotypic heterogeneity of many sorts is typical in spatially structured microbial populations such as biofilms [7]. Hence, models and robust and efficient numerical methods of the sort presented here are likely to be useful and possibly necessary for modeling the function and ecology of diverse, spatially unmixed microbial populations that dominate the natural environment.

Acknowledgment. The authors thank Phil Stewart for helpful ideas and discussions. BPA was supported by the National Science Foundation under Grant No. DMS-0914514. IK was supported by the National Science Foundation under Grant Nos. DMS-0934696 and DMS-1022836. 


\section{REFERENCES}

[1] E. Alpkvist and I. Klapper, A multidimensional multispecies continuum model for heterogeneous biofilm development, Bull. Math. Biol., 69(2), 765-789, 2007.

[2] B.P. Ayati, A variable time step method for an age-dependent population model with nonlinear diffusion, SIAM J. Numer. Anal., 37(5), 1571-1589, 2000.

[3] B.P. Ayati and T.F. Dupont, Galerkin methods in age and space for a population model with nonlinear diffusion, SIAM J. Numer. Anal., 40(3), 1064-1076, 2002.

[4] B.P. Ayati and T.F. Dupont, Convergence of a step-doubling Galerkin method for parabolic problems, Math. Comput., 74(251), 1053-1065, July 2005.

[5] B.P. Ayati and T.F. Dupont, Mollified birth in natural-age-grid Galerkin methods for agestructured biological systems, Nonlinearity, 22(8), 1983-1995, 2009.

[6] B.P. Ayati and I. Klapper, A multiscale model of biofilm as a senescence-structured fluid, Multiscale Model. Simul., 6(2), 347-365, 2007.

[7] B.R. Boles, M. Thoendel, and P. K. Singh, Self-generated diversity produces "insurance effects" in biofilm communities, PNAS, 101(47), 16630-16635, 2004.

[8] L.E. Chávez de Paz, I.R. Hamilton, and G. Svensäter, Oral bacteria in biofilms exhibit slow reactivation from nutrient deprivation, Microbiology, 154, 1927-1938, 2008.

[9] N.G. Cogan, Effects of persister formation on bacterial response to dosing, J. Theor. Biol., 238(3), 694-703, 2006.

[10] N.G. Cogan, Incorporating toxin hypothesis into a mathematical model of persister formation and dynamics, J. Theor. Biol., 248(2), 340-349, 2007.

[11] A.M. de Roos, Numerical methods for structured population models: The escalator boxcar train, Num. Meth. Part. Diff. Eqns., 4, 173-195, 1989.

[12] A.M. de Roos, A gentle introduction to physiologically structured population models, in S. Tuljapurkar and H. Caswell, editors, Structured-population Models in Marine, Terrestrial, and Freshwater Systems, Population and Community Biology Series, Chapman \& Hall, New York, 18(5), 119-204, 1997.

[13] J. Dockery and I. Klapper, Finger formation in biofilm layers, SIAM J. Appl. Math., 62(3), 853-869, 2001.

[14] W. Gujer and O. Wanner, Biofilms, Chapter Modeling Mixed Population Biofilms, John Wiley \& Sons, 397-443, 1990.

[15] I. Klapper and B. Szomolay, An exclusion principle and the importance of mobility for a class of biofilm models, Bull. Math. Biol., 73(9), 2213-2230, 2011.

[16] I. Klapper and J. Dockery, Mathematical description of microbial biofilms, SIAM Rev., 52(2), 221-265, 2010.

[17] I. Klapper, P. Gilbert, B.P. Ayati, J. Dockery, and P. Stewart, Senescence can explain microbial persistence, Microbiology, 153(11), 3623-3630, 2007.

[18] T. Malik and H.L. Smith, A resource-based model of microbial quiescence, J. Math. Biol., 53(2), 231-252, 2006.

[19] T. Malik and H.L. Smith, Does dormancy increase fitness of bacterial populations in timevarying environments? Bull. Math. Biol., 70(4), 1140-1162, 2008.

[20] J. Roostalu, A. Jõers, H. Luidalepp, N. Kaldalu, and T. Tenson, Cell division, in Escherichia colicultures monitored at single cell resolution, BMC Microbiology, 8(68), 1-14, 2008.

[21] H.L. Smith and P. Waltman, The Theory of the Chemostat, in Cambridge Series in Mathematical Biology, Cambridge University Press, 13, 1995.

[22] P.S. Stewart and M.J. Franklin, Physiological heterogeneity in biofilms, Nature Reviews Microbiology, 6(3), 199-210, 2008.

[23] D. Sulsky, Numerical solution of structured population models, II. mass structure, J. Math. Biol., 32, 491-514, 1994.

[24] O. Wanner, H. Eberl, E. Morgenroth, D. Noguera, C. Picioreanu, B. Rittmann, and M. Van Loosdrecht, Mathematical Modeling of Biofilms, in IWA Scientific and Technical Reports, IWA Publishing, London, 18, 2006.

[25] G.F. Webb, Population models structured by age, size, and spatial position, in Structured Population Models in Biology and Epidemiology, Springer-Verlag, 2008.

[26] T.C. Zhang, Y.C. Fu, and P.L. Bishop, Competition in biofilms, Wat. Sci. Tech., 29(10-11), 263-270, 1994. 